Review Article

\title{
A REVIEW ON BIOACTIVE PHYTOCHEMICALS AND IT'S MECHANISM ON CANCER TREATMENT AND PREVENTION BY TARGETING MULTIPLE CELLULAR SIGNALING PATHWAYS
}

\author{
VARSHA L. BHUTADIYA1, KINNARI N. MISTRY1* \\ Ashok and Rita Patel Institute of integrated study and research in Biotechnology and Allied Science (ARIBAS), New Vidyanagar 388121, \\ Gujarat, India \\ *Email: kinnarimistry@aribas.edu.in
}

Received: 20 Jun 2021, Revised and Accepted: 26 Oct 2021

\begin{abstract}
In developing and developed countries, cancer is a significant health problem in people. Cancer becomes the second greatest cause of death in human after cardiovascular disease. However, significant advancements in modern cancer therapies have a beneficial impact on survival, chemotherapy and radiation therapy. Plants fulfill our basic needs to continue life and provide natural products that help to cure disease. The medicinal plants are readily available and have no toxicity as compared to modern drugs. Phytochemicals act on metabolic pathways and inhibit tumor growth, the development of cancerous cells, and replication by different mechanisms. Apigenin's chemo-preventive and anticancer activities have been demonstrating in numerous studies. Curcumin is a polyphenolic compound isolated from the Curcuma longa plant. EGCG, a polyphenol in black, white, and green tea is a chemo-preventive effect against many cancers by targeting multiple pathways. Normal cell growth and cell proliferation are closely regulated processes. The JAK-STAT (Janus kinase-signal transducer and activator of transcription) pathway controls gene expression during different processes, including proliferation, initiation, and apoptosis. The transcription factors are associated with the growth of cancer cells and control a cellular function in the disease. Mitogen-activated protein kinase (MAPK) is a class of serine and threonine kinase that includes ERK (extracellular regulated kinase), JNK (c-Jun N-terminal kinases), and p38. This review paper describes natural phytochemical compounds, their molecular targets and mechanisms of action.
\end{abstract}

Keywords: Cancer, Medicinal plants, Phytochemicals, Signaling pathways

(C) 2021 The Authors. Published by Innovare Academic Sciences Pvt Ltd. This is an open access article under the CC BY license (https://creativecommons.org/licenses/by/4.0/) DOI: https://dx.doi.org/10.22159/ijpps.2021v13i12.42798. Journal homepage: https://innovareacademics.in/journals/index.php/ijpps.

\section{INTRODUCTION}

Hippocrates, a Greek physician, was the first to name cancer, combining the Greek words "carcinoma" and "cancer." The term "karakinos" is used for a tumor. Cancer comes to be the second most significant source of death in humans after cardiovascular disease. In both developed and developing countries, cancer is an unusual common health complication. Every year, an estimated 10.9 million new cancer cases, 6.7 million deaths, and 24.6 million human living with different types of cancer globally [1]. Because of late observation and low treatment effectiveness, cancer has developed resistance to the chemotherapeutic drug and unsatisfactory treatment. Radiation therapy or chemotherapy causes side effects like sickness, exhaustion, hair loss, vomiting, depression. It also affects the immunological system, kidney, urinary tract, gastrointestinal organs, neurologic and psychological changes [2]

Plants fulfill our basic needs to continue life and provide natural products that help in curing disease. In the traditional system like Ayurveda, Unani plants are used as medicines for primary health care and provide many novel compounds used for preventing and curative treatment to modern science [3]. In addition, researchers in developing countries have discovered plant species with anticancer properties that have been used in herbal medicine. Moreover, medicinal plants are less expensive than current drugs and have no toxic effect [4].

Compounds isolated from plants play a different role in cancer medication; for example, Epipodophyllotoxin, camptothecin, combretastatin, taxol and vinca alkaloids. Vindesine (VDS) and vinorelbine (VRLB) are vinca alkaloids that are semi-synthetic analogs. The vinblastine, vincristine, and vinca alkaloids are chemotherapeutic drugs, were isolated from Catharanthus roses [5].

Natural phytochemicals used as cancer chemo-inhibitory and therapeutic substance

Phytochemicals are active compounds isolated from various medicinal plants that have been found to have chemotherapeutic effects on cancer cell lines. Phytochemicals act on metabolic pathways and inhibit tumor growth, cancer cell development, and replication through various mechanisms [6].

\section{Apigenin}

Apigenin is a flavonoid compound that naturally originates in many plants and helps suppress HeLa cells multiplication. Apigenin has chemo-preventive and anti-cancerous properties. The ERK/AP-1/COX-2 pathway was inhibited by apigenin, which inhibited COX-2 activity in PMA-induced breast cancer cells. Apigenin suppresses Helicobacter pylori-induced inflammation. It promotes IKB expression in gastric cancer cells while suppressing COX-2 and NF-kB activity [7]. In prostate cancer cells of human, apigenin decreased the activity of NF-kB and its responsive genes. Apigenin inhibited the PGE2 level in colon cancer cells. Apigenin disrupts the leptin/leptin receptor pathway in a non-small cell lung cancer cell line, resulting in cell death [8].

\section{Berberine}

Berberine is a plant-derived alkaloid with anticancer and chemopreventive properties. Clinical experiments for different disorders, including skin, intestine, breast, and oral cancers, are ongoing. Berberine inhibits several signaling cascades in colon cancer cells, including STAT3 and MMP2/MMP-9 [9]. COX and mTOR (mammalian target of rapamycin) pathways blocked by berberine. The various functions of berberine are arresting the cell cycle, inducing the apoptosis pathway, inhibiting the NF-kB pathway, and repressed COX-2 expression at the mRNA and protein level in colon cancer cells (HT-29). Berberine targets NF-kB/COX-2 and activates the apoptosis pathway in oral cancer cells [10]. In melanoma cells, berberine induces the expression of AMPK and inhibiting the ERK signaling pathways and COX-2 expression [11].

\section{Curcumin}

Curcumin is a polyphenolic compound originate from the Curcuma longa plant (1, 7-bis [4-hydroxy-3-methoxyphenyl]-1E, 
6Eheptadiene-3, 5-dione). Curcumin suppresses tumor cell development by controlling different cell signaling pathways, including tumor suppressor pathway (p21, RB, p53), caspase activation (caspase-8, 3, 9), cell cycle (cyclin D1 expression), mitochondrial pathway of apoptosis, extrinsic apoptosis pathway, PI3-K/Akt cell survival pathway, and mitogen-activated protein kinase pathway [12]. In lung cancer cells, curcumin hinders the IFNinduced activation of COX-2 and NF-kB activity. Curcumin inhibits IL-6, IL-1, and other pro-inflammatory mediators through an NF-kB, AP-1, and MAPK dependent pathway. Curcumin was discovered to have anti-inflammatory properties. In human prostate cancer cells, the proteins of Akt and mTOR pathways, their downstream targets, are phosphorylated by curcumin [13].

\section{Epigallocatechin-3-gallate}

EGCG is a polyphenolic compound in green tea; it is also found in small amounts in white and black teas. It has a chemo-preventive activity against different cancer by targeting multiple pathways. Anti-cancer effect of EGCG shown in various cancer like brain, prostate, cervical, and bladder [14]. In vitro, EGCG prevented skin cancer cells. EGCG slows down the Bcl-xL and its antiapoptotic protein activity. COX-2 is repressed by EGCG, which inhibits by blocking the PI3K/AKT pathway. EGCG inhibits its gene products such as VEGEF and c-Myc. EGCG suppressed cell emigration in melanoma cells [15].

\section{Fisetin}

Fisetin is present in onions, strawberries, persimmons, apples, and cucumbers. It is an anti-inflammatory compound with anticancer effects. Fisetin prevents carcinogenesis in HCT-116 cells [16]. fisetin inhibits the COX-2/iNOS and NF-kB signaling pathways in skin cancer. Fisetin inhibited adherence, emigration, and aggression by lowering the amount of nuclear factor kappa B (NF-kB) and activator protein-1 (AP-1). Fisetin inhibited cell viability by causing arrest in the G1-phase of the cell cycle and disrupting Wnt/catenin signaling in lung cancer cells [17].

\section{Genistein}

Fava beans, coffee, lupine, kudzu, and soybeans contain genistein, a form of isoflavone. Antiangiogenic, antioxidant, and anthelmintic properties have been discovering in genistein. Genistein inhibits DNA topoisomerase II activity and interrupts the activity of the tyrosine kinase enzyme [18]. Genistein has been indicating to be involved in the medication of leukemia in in vivo and in vitro research. Genistein induces the apoptosis pathway and inactivates NF-kB in a lung cancer cell line (H460). In addition, genistein's activation of the cell cycle is interrupted in breast cancer cells [19].

\section{Kaempferol}

Brussels sprouts, broccoli, grapefruit, witch hazel, tea, apples, and other plants contain the flavonoid kaempferol. It has been used in the medication for lung and pancreas cancer. It has been studied for its antiangiogenic activity, antitumor, and free-radical scavenging properties. Kaempferol is a flavonoid with antitumor, antioxidant properties, and also capacity to cause apoptosis in cancer cells [20]. Kaempferol inhibits CDK1 and thus prevents the G2/M stage of the cell cycle in human breast cancer cells. In MCF-7 cells, kaempferol effectively induced PARP cleavage, accompanied by a reduction in Bcl2 protein expression and multiplied in Bax protein expression [21]. As an aryl hydrocarbon receptor (AhR) blocker, kaempferol inhibits the PI3K-Akt pathway, which may be helpful in the treatment of esophageal cancer [22].

\section{Lycopene}

Lycopene is a carotenoid with anti-cancer properties and can be found in onions, red carrots, red papayas, and watermelons. Foods rich in lycopene, such as tomatoes, can slow down the possibility of prostate cancer. Lycopene has been appearing to have antioxidant and chemo-inhibitory effects in prostate cancer. Lycopene is poorly soluble in water but highly soluble in organic solvents. However, cancer-preventive enzymes, including phase II detoxification enzymes, are responsible for their anti-cancer properties. Lycopene has been appearing to have antioxidant and antimutagenic activity. In addition, lycopene protects the body from chemical toxicity [23].

\section{Quercetin}

Quercetin is a flavonoid. It is found in licorice leaves and fruits that have anti-cancer, anti-inflammatory, and polyphenol properties. Quercetin has anti-estrogenic effects, which slow down metastasis of breast cancer cells [24]. Quercetin controls cancerous cell development by stimulating tumor suppressor genes, initiating apoptosis pathways, and slowing down angiogenesis. Quercetin inhibited proliferation, reduced PGE2 levels, and increased apoptosis in HCA-7 human colon cancer cells during colon carcinogenesis [25].

\section{Resveratrol}

Resveratrol, a polyphenolic molecule, is found in grapes, peanuts, red wine, dark berries, and other plants [26]. Many research studies conducted over the previous decade have appeared that resveratrol can hinder or delay the enlargement of disorders like cancer. It has chemoprevention capacity against many stages of cancers, such as beginning, development and progression. Resveratrol caused COX-2 intranuclear aggregation in breast cancer cells and allowed COX2 with Ser15-phosphorylated p53. In colon epithelial cells (HT-29), resveratrol suppressed the synthesis of Prostaglandin E2 (PGE2) by inhibiting COX-2 enzyme activity [27]. Resveratrol inhibits NF-kB function, blocks tumor necrosis factor, increases COX-2 activity and increases the immunoregulatory cytokines in liver cells. Resveratrol suppresses the development of breast cancer by inhibiting IkB kinase/NF-kB signaling and induces the expression of COX-2. Resveratrol also activates the tumor suppressor gene p53 [28].

\section{Ursolic acid}

Ursolic acid, a natural triterpenoid compound found in plants, is being investigated as a cancer treatment agent. Ursolic acid stops cancer from spreading by blocking many signaling pathways [29]. For example, ursolic acid downregulates COX-2 activity and cleaved poly (ADP-ribose)-polymerase, which activates the apoptosis pathway in liver cancer cells (HepG2) [30]. In addition, ursolic acid inhibits cellular growth, increases apoptosis, and stops the cell cycle in gastric cancer cells [31].

Molecular-level attack for natural chemo-inhibitory substances Apoptosis

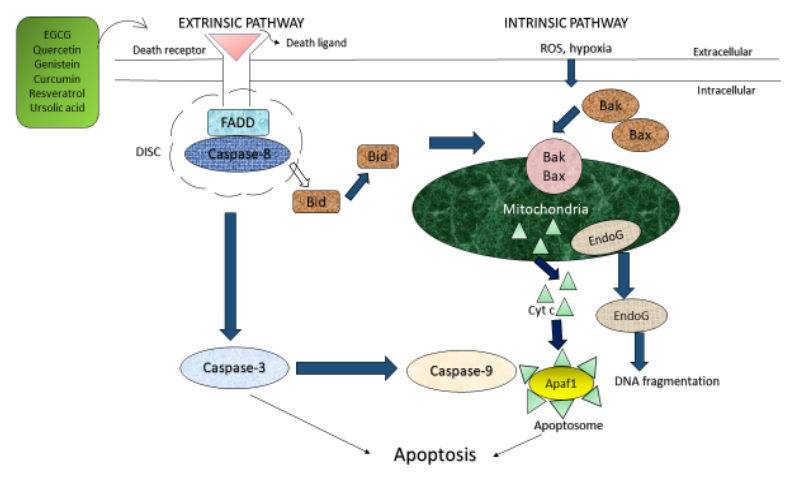

Fig. 1: In various cancers, phytochemicals activate the mechanism of the apoptosis pathway

The two most general types of cell death are necrosis and apoptosis. External damage, the disappearing of the plasma membrane or its biochemical supports causes necrosis or cell death. Uncontrollable cell development and decreased apoptosis are a marker of cancer. Programmed cell death via apoptosis cascade is necessary for the proper development of multicellular organisms. The morphological and biochemical changes in the apoptosis pathway are chromatin condensation, inter nucleosomal DNA fragmentation, the origination of apoptotic bodies, and cell shrinkage [32]. Apoptosis can destroy genetically modified, pre-cancerous, or cancerous cells. Several 
natural plant compounds were originally developed as antiviral or anti-inflammatory agents. Still, as our perception of cancer mechanisms improves, the antitumor properties of different phytochemicals are becoming more commonly accepted and used [33]. In various cancer cells, EGCG can increase the apoptosis pathway and inhibit the cell cycle. These effects of EGCG in cell lines were in millimolar and micromolar concentrations [34]. Quercetin prevents cellular proliferation by inducing the intrinsic pathway of apoptosis in different cancer. It enhanced the activity of the pro-apoptotic Bcl-2 class of proteins and triggered caspase- 9 and caspase- 3 but not caspase-8 [35]. Red wine contains resveratrol, which is an antioxidant. It also decreased the activity of antiapoptotic protein $\mathrm{Bcl}-2$ while increasing the pro-apoptotic Bcl-2 class. The Bcl-2 class contains Bid, Bax, Bad, and Bak proteins. In BGC-803 cancer cells, ursolic acid promotes DNA fragmentation, upregulating caspase3,-8, and-9 and downregulating Bcl-2 expression [fig. 1] [36].

\section{Cell cycle}

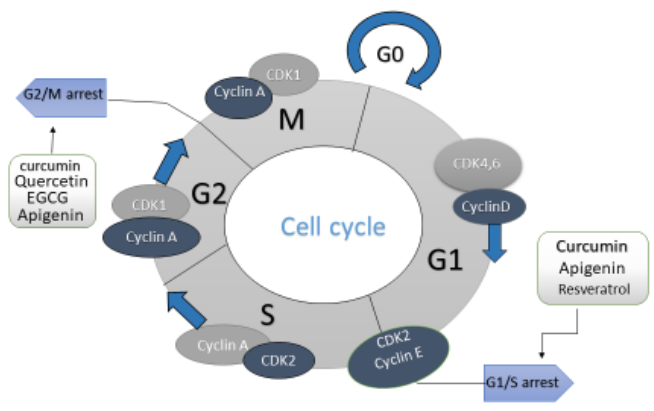

Fig. 2: Mechanisms of cell cycle prevent initiate by phytochemicals in different cancer

Cell growth and proliferation are tightly controlled processes in normal cells, and cell cycle dysregulation can result in unregulated cell proliferation and contribute to the malignant phenotype of tumor cells [37]. Cancer cells lack cell cycle regulation. Cell cycle checkpoints regulate cell growth, differentiation of cells, DNA replication, and apoptosis. Many phytochemicals have been occurring to hinder cancer cell development by controlling cell cycle proteins. Additionally, cell cycle checkpoints G1/S and G2/M are essential targets in cell cycle regulation [38].

Curcumin caused G2/M and G1/S phase in HOS cells to prevent D1 cyclin, cdc2, and cyclin B1 downregulation. Quercetin arrests G2/M by upregulating p73, p21 and waf1 while downregulating cyclin B1 in a human cancer cell line. Resveratrol promotes cell cycle inhibition and aggregation of cells in the G0-G1 phase in Pancreatic cancer cells. Resveratrol arrests cell cycle in G2-M and S phases and degradation in pancreatic cancer cells [39]. Apigenin inhibits Huh7 cell development, initiates the G2/M cell cycle prevention, and increases the rate of the apoptosis pathway. Apigenin prevented the enlargement of leukemia cells by allowing them to join the G2/M phase (myeloid HL60 cells) and the G0/G1 phase (non-myeloid HL60 cells, erythroid TF1 cells). In multiple myeloma cells, isothiocyanates promote cell cycle prevention in the G2/M phase and phosphorylation of conserved serine residue in the histone H3 [40].

\section{JAK-STAT pathway}

The JAK-STAT (Janus kinase, signal transducer, and activator of transcription) pathway controls gene expression during initiation, enlargement, migration, and apoptosis [41]. STAT proteins were originally identified as cytoplasmic transcription factors, only translocated to the nucleus upon Jak-mediated phosphorylation and dimerization. When epidermal growth factors and cytokines interact, they generate homodimers or heterodimers that move to the nucleus of the cell and regulate genes [42]. The anti-apoptotic and proliferative properties of STAT3 in cancer cells have been connected to a poor prognosis. Stat3 dysregulation has been connected to increased breast cancer cell enlargement, survival, and metastasis. In myeloma, the Bcl-xL and Mcl-1 are anti-apoptotic proteins that were overexpressed in myeloma cells where integral Stat3 activity was promoted by Interleukins [43]. By reducing VEGF and Stat 3 activation, epigallocatechin gallate reduces gastric cancer origination and angiogenesis. In hypopharynx carcinoma and breast cancer cells in human, epigallocatechin gallate slows down Stat3 stimulation [44]. $\mathrm{Cu} \quad(2+)$-oxidized LDL-induced endothelial apoptosis was reduced by quercetin through the Jak2-Stat3 pathways. In vitro treatment of activated $\mathrm{T}$ cells with quercetin inhibited IL-12-induced tyrosine phosphorylation of Jak2, Tyk2, Stat3, and Stat4, which results in IL-12-induced T cell proliferation and Th1 differentiation [fig. 3] [45].

\section{MAPK pathway}

Mitogen-activated protein kinase (MAPK) is particular to serine/threonine kinases that include JNK, ERK (extracellular regulated kinase), and p38. They control several processes like cell development and enlargement of cells by transferring cell signals to the nucleus. Transmitting cell signals to the nucleus affects gene expression that controls cell processes like cell development, differentiation, and multiplication [46]. The epidermal growth factor receptor (EGFR), estrogen receptor (ER), and tumor necrosis factoralpha (TNF) are the most considerable downstream effectors. Components of MAPK pathways, for example, PI3K, Ras, and Akt, are mutated in human cancer. Raf-mediated chemotherapeutic drug resistance induces paclitaxel in breast cancer cells. B-Raf has been occurring to evolve in cancer such as thyroid and breast cancer. Curcumin down regulates EZH2 by activating three important members of the mitogen-activated protein kinase (MAPK) pathway: c-Jun NH2-terminal kinase (JNK), extracellular signal-regulated kinase (ERK), and p38 kinase [47]. Ursolic acid suppresses the EGFR/MAPK pathway, slows down the enlargement of colon cancer cells, and activates the apoptosis pathway. In osteosarcoma cells, ursolic acid activates the MAP kinase pathway, inducing caspasedependent apoptosis, significantly inhibited by ERK1/2 inhibitor treatment [fig. 3] [48].

\section{NF-kB pathway}

The transcription factors are related to cancer growth and control a different cellular cascade in the disease. NF-kB inducers include tumor necrosis factor-alpha, lipopolysaccharide, reactive oxygen species (ROS), and Interleukin beta [49]. Rel/NF-kB transcription complexes are found in an inactivated state in the cytoplasm of most cells, where they are attached to an inhibitor (IkB). Many stimuli can rapidly activate these transcription complexes from their inhibitors and allowing them to translocate to the nucleus [50]. The transcription factor NF-kB controls the expression of genes impenetrable in cancer invasion, angiogenesis, and metastasis. As an effect, inhibitors of NF-kB activity have been occurring to be helpful in cancer medication [51].

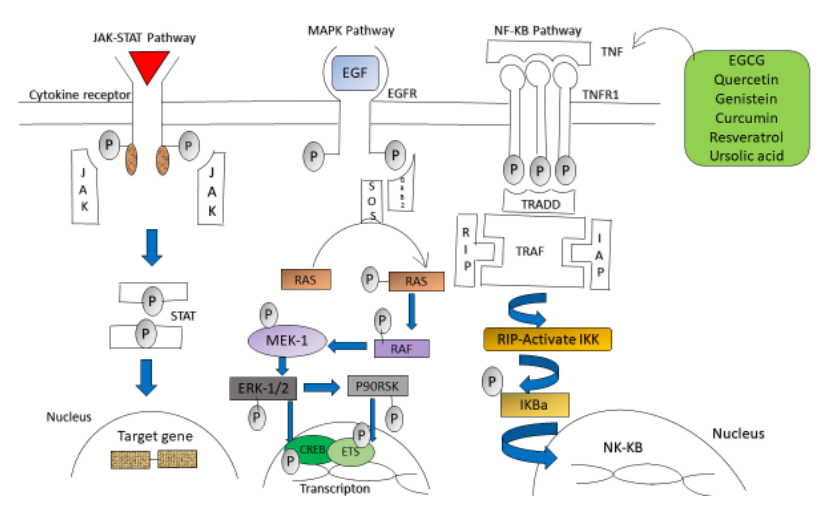

Fig. 3: Effect of phytochemicals on signaling pathway in different cancer

Resveratrol suppressed the NF-kB-regulated gene, inhibiting apoptosis and preventing caspase-3 activation. Tumor necrosis factor promotes phosphorylation of the NF-kB. Resveratrol inhibits 
TNF-promoted phosphorylation and nuclear transfer of the NF-kB p65 subunit [52]. Curcumin inhibits the transcription factor NF-kB and slows down the phosphorylation of IKB alpha. NF-kB initiated the apoptosis pathway and repressed the IKB kinase, preventing proliferation [fig. 3] [53].

\section{CONCLUSION}

Major cancer therapeutics are radiation therapy and chemotherapy, which causes severe side effects such as hepatic toxicity, nausea, vomiting, etc. Natural plant products can lower the toxicity of radiation therapy and chemotherapy. This review gives information about different phytochemicals and their anticancer activity by targeting apoptosis, cell cycle, JAK-STAT, MAPKs, and NF-kB activation in vitro research.

\section{ACKNOWLEDGMENT}

The authors are grateful to Charutar Vidya Mandal (CVM) Vallabh Vidyanagar, Gujarat, for providing the platform for this research work and SHODH-ScHeme of Developing High-quality research Education Department, Gujarat State, for providing fund.

\section{ABBREVIATIONS}

JAK-STAT-Janus kinase-signal transducer and activator of transcription, MAPKMitogen-activated protein kinase, ERKextracellular regulated kinase, JNK-c-Jun N-terminal kinases, VDSVindesine, VRLB-vinorelbine, NF-kB-nuclear factor-kappa B, AP-1activator protein-1, AhR-aryl hydrocarbon receptor, EGFR-The epidermal growth factor receptor, ER-estrogen receptor, TNF-tumor necrosis factor-alpha, ERK-extracellular regulated kinase, EZH2zeste homolog 2, ROS-reactive oxygen species

\section{FUNDING}

Nil

\section{AUTHORS CONTRIBUTIONS}

All the authors have contributed equally.

\section{CONFLICT OF INTERESTS}

The authors declared that they have no conflict of interest.

\section{REFERENCES}

1. Sudha M. Anti cancer herbs used in Siddha system of medicine: a review. IJPPR. 2018;12:118-34.

2. Chanchal Dilip Kumar. Various medicinal plants used in the treatment of anticancer activity. Int J Pharm Sci. 2017;9:1424-9.

3. Sharma Deepika, Dangi DCBS. Role of plants in treating cancer: a review. Int J Adv Res. 2016;4(7):901-7. doi: 10.21474/IJAR01/969.

4. Adhikari Partha Pradip, Paul Satya Bhusan. Medicinally important plant cleome gynandra: A phytochemical and pharmacological explanation. Asian J Pharm Clin Res. 2018;11(1):21-6. doi: 10.22159/ajpcr.2017.v11i1.22037.

5. Cragg Gordon M, Newman David J. Plants as a source of anticancer agents. J Ethnopharmacol. 2005;100(1-2):72-9. doi: 10.1016/j.jep.2005.05.011, PMID 16009521.

6. Balabhaskar R, Rajendra Kumar A, Selvarajan S, Faridha A, Gayathri Gunalan. Potential natural products with anticancer properties and their applications. Asian J Pharm Clin Res. 2019;12:27-33. doi: 10.22159/ajpcr.2019.v12i5.32817.

7. Wang Yuan-Chuen, Huang Kai-Ming. In vitro anti-inflammatory effect of apigenin in the Helicobacter pylori-infected gastric adenocarcinoma cells. Food Chem Toxicol. 2013;53:376-83. doi: 10.1016/j.fct.2012.12.018, PMID 23266501.

8. Bruno Andreina, Siena Liboria, Gerbino Stefania, Ferraro Maria, Chanez Pascal, Giammanco Marco, Gjomarkaj Mark, Pace Elisabetta. Apigenin affects leptin/leptin receptor pathway and induces cell apoptosis in lung adenocarcinoma cell line. Eur J Cancer. 2011;47(13):2042-51. doi: 10.1016/j.ejca.2011.03.034, PMID 21550230.

9. Liu Xuan, Ji Qing, Ye Naijing, Sui Hua, Zhou Lihong, Zhu Huirong, Fan Zhongze, Cai Jianfeng, Li Q. Berberine inhibits invasion and metastasis of colorectal cancer cells via COX-
2/PGE 2 mediated JAK2/STAT3 signaling pathway. PLOS ONE. 2015;10(5):e0123478.

10.1371/journal.pone.0123478, PMID 25954974.

10. Tai WP, Luo HS. The inhibit effect of berberine on human colon cell line cyclooxygenase-2. Zhonghua Nei $\mathrm{Ke} \quad \mathrm{Za}$ Zhi. 2003;42(8):558-60. PMID 14505547.

11. Kim Hak Su, Kim Myung Jin, Kim Eun Ju, Yang Young, Lee Myeong-Sok, Lim Jong-Seok. Berberine-induced AMPK activation inhibits the metastatic potential of melanoma cells via reduction of ERK activity and $\mathrm{COX}-2$ protein expression. Biochem Pharmacol. 2012;83(3):385-94. doi: 10.1016/j.bcp.2011.11.008, PMID 22120676.

12. Krisnamurti Desak Gede Budi, Wanandi Septelia Inawati, Louisa Melva. Curcumin increases the sensitivity of breast cancer cells to tamoxifen by inhibiting MRP2 mrna expression of efflux transporter MRP2. Int J Appl Pharm. 2019;11:88-90. doi: 10.22159/ijap.2019.v11s6.33553.

13. Yu Siwang, Shen Guoxiang, Khor Tin Oo, Kim Jung Hwan, Kong Ah-Ng Tony. Curcumin inhibits Akt/mammalian target of rapamycin signaling through protein phosphatase-dependent mechanism. Mol Cancer Ther. 2008;7(9):2609-20. doi: 10.1158/1535-7163.MCT-07-2400, PMID 18790744.

14. Harper Curt E, Patel Brijesh B, Wang Jun, Eltoum Isam A, Lamartiniere Coral A. Epigallocatechin-3-gallate suppresses early stage, but not late-stage prostate cancer in TRAMP mice: mechanisms of action. Prostate. 2007;67(14):1576-89. doi: 10.1002/pros.20643, PMID 17705241.

15. Singh Tripti, Katiyar Santosh K. Green tea polyphenol,(-)epigallocatechin-3-gallate, induces toxicity in human skin cancer cells by targeting $\beta$-catenin signaling. Toxicol Appl Pharmacol. 2013;273(2):418-24. 10.1016/j.taap.2013.09.021, PMID 24096034.

16. Lim Do Y, Park Jung Han Yoon. Induction of p53 contributes to the apoptosis of HCT-116 human colon cancer cells induced by the dietary compound fisetin. Am J Physiol Gastrointest Liver Physiol. 2009;296(5):G1060-8. doi: 10.1152/ajpgi.90490.2008, PMID 19264955.

17. Liao Yi-Chen, Shih Yuan-Wei, Chao Chang-Hung, Lee Xin-Yan, Chiang Tai-An. Involvement of the ERK signaling pathway in fisetin reduces invasion and migration in the human lung cancer cell line A549. J Agric Food Chem. 2009;57(19):8933-41. doi: 10.1021/jf902630w, PMID 19725538.

18. Lopez Lazaro Miguel, Willmore Elaine, Austin Caroline A. Cells lacking DNA topoisomerase II $\beta$ are resistant to genistein. J Nat Prod. 2007;70(5):763-7. doi: 10.1021/np060609z, PMID 17411092.

19. Magee Pamela J, Rowland Ian R. Phyto-oestrogens, their mechanism of action: current evidence for a role in breast and prostate cancer. Br J Nutr. 2004;91(4):513-31. doi: 10.1079/BJN20031075, PMID 15035679.

20. Gacche Rajesh N, Shegokar Harshala D, Gond Dhananjay S, Yang Zhenzhou, Jadhav Archana D. Evaluation of selected flavonoids as antiangiogenic, anticancer, and radical scavenging agents: an experimental and in silico analysis. Cell Biochem Biophys. 2011;61(3):651-63. doi: 10.1007/s12013-011-9251z, PMID 21830125.

21. Yi Xiaofang, Zuo Jiangcheng, Tan Chao, Xian Sheng, Luo Chunhua, Chen Sai, Yu Liangfang, Luo Yucheng. Kaempferol, a flavonoid compound from Gynura medica induced apoptosis and growth inhibition in mcf-7 breast cancer cell. Afr J Tradit Complement Altern Med. 2016;13(4):210-5. doi: 10.21010/ajtcam.v13i4.27, PMID 28852738.

22. To Kenneth KW, Yu L, Liu Shuwen, Fu Jianhua, Cho Chi Hin. Constitutive AhR activation leads to concomitant ABCG2-mediated multidrug resistance in cisplatin-resistant esophageal carcinoma cells. Mol Carcinog. 2012;51(6):449-64. doi: 10.1002/mc.20810, PMID 21678497.

23. Giovannucci Edward, Ascherio A, Rimm EB, Stampfer MJ, Colditz GA, Willett WC. Intake of carotenoids and retino in relation to risk of prostate cancer.J Natl Cancer Inst. 1995;87(23):1767-76. doi: 10.1093/jnci/87.23.1767, PMID 7473833.

24. Wang Hongxian, Tao Linyu, Qi K, Zhang Haoyun, Feng Duo, Wei Wenjun, Kong Heng, Chen Tianwen, Lin Qiusheng. Quercetin 
reverses tamoxifen resistance in breast cancer cells. J BUON. 2015;20(3):707-13. PMID 26214621.

25. Al-Fayez Mohammad, Cai Hong, Tunstall Richard, Steward William P, Gescher Andreas J. Differential modulation of cyclooxygenase-mediated prostaglandin production by the putative cancer chemopreventive flavonoids tricin, apigenin and quercetin. Cancer Chemother Pharmacol. 2006;58(6):81625. doi: 10.1007/s00280-006-0228-3, PMID 16552572.

26. Bishayee Anupam. Cancer prevention and treatment with resveratrol: from rodent studies to clinical trials. Cancer Prev Res (Phila). 2009;2(5):409-18. doi: 10.1158/1940-6207.CAPR08-0160, PMID 19401532.

27. Zykova Tatyana A, Zhu Feng, Zhai Xiuhong, Ma Wei-Ya, Ermakova Svetlana P, Lee Ki Won, Bode Ann M, Dong Zigang. Resveratrol directly targets COX-2 to inhibit carcinogenesis. Molecular carcinogenesis: published in cooperation with the University of Texas M D Anderson Cancer Center. 2008;10:797-805.

28. Park, Sin Aye, Hye Kyung Na, Young Joon Surh. Resveratrol suppresses 4-hydroxyestradiol-induced transformation of human breast epithelial cells by blocking ІкB kinase $\beta$-NF- $\kappa B$ signalling. Free Radical Res. 2012;8:1051-7.

29. Shanmugam Muthu K, Dai Xiaoyun, Kumar Alan Prem, Tan Benny KH, Sethi Gautam, Bishayee Anupam. Ursolic acid in cancer prevention and treatment: molecular targets, pharmacokinetics and clinical studies. Biochem Pharmacol. 2013;85(11):1579-87. 10.1016/j.bcp.2013.03.006, PMID 23499879.

30. Iqbal Javed, Abbasi Banzeer Ahsan, Ahmad Riaz, Mahmood Tariq, Kanwal Sobia, Ali Barkat, Khalil Ali Talha, Shah Sayed Afzal, Alam Muhammad Maqsood, Badshah Hussain. Ursolic acid a promising candidate in the therapeutics of breast cancer: current status and future implications. Biomed Pharmacother. 2018;108:752-6. doi: 10.1016/ j.biopha.2018.09.096, PMID 30248543.

31. Zhang Yi-Ying, Deng Tao, Hu Zhi-Fang, Zhang Qiu-Ping, Zhang Jing, Jiang Hua. Mechanisms of inhibiting proliferation and inducing apoptosis of human gastric cancer cell line SGC7901 by ursolic acid. Ai Zheng. 2006;25(4):432-7. PMID 16613675.

32. D’Agostini Francesco, Izzotti Alberto, Balansky Roumen M, Bennicelli Carlo, De Flora Silvio. Modulation of apoptosis by cancer chemopreventive agents. Mutat Res. 2005;591(12):173-86. doi: 10.1016/j.mrfmmm.2005.03.034, PMID 16137721.

33. Li-Weber Min. Targeting apoptosis pathways in cancer by Chinese medicine. Cancer Lett. 2013;332(2):304-12. doi: 10.1016/j.canlet.2010.07.015, PMID 20685036.

34. Khan Naghma, Mukhtar Hasan. Multitargeted therapy of cancer by green tea polyphenols. Cancer Lett. 2008;269(2):269-80. doi: 10.1016/j.canlet.2008.04.014, PMID 18501505.

35. Murakami Akira, Ashida Hitoshi, Terao Junji. Multitargeted cancer prevention by quercetin. Cancer Lett. 2008;269(2):31525. doi: 10.1016/j.canlet.2008.03.046, PMID 18467024.

36. Manu KA, Kuttan Girija. Ursolic acid induces apoptosis by activating p53 and caspase-3 gene expressions and suppressing NF-kappaB mediated activation of bcl-2 in B16F10 melanoma cells. Int Immunopharmacol. 2008;8(7):974-81. doi: 10.1016/j.intimp.2008.02.013, PMID 18486908.

37. Rezaei Parisa Fathi, Fouladdel Shamileh, Ghaffari Seyed Mahmood, Amin Gholamreza, Azizi Ebrahim. Induction of G1 cell cycle arrest and cyclin D1 down-regulation in response to pericarp extract of Baneh in human breast cancer T47D cells. Daru. 2012;20(1):101. doi: 10.1186/2008-2231-20-101, PMID 23351343.

38. Araujo Joao R, Gonçalves Pedro, Martel Fatima. Chemopreventive effect of dietary polyphenols in colorectal cancer cell lines. Nutr Res. 2011;31(2):77-87. doi: 10.1016/j.nutres.2011.01.006, PMID 21419311.

39. Cui Jing, Sun Renhu, Yu Yangping, Gou Shanmiao, Zhao Gang, Wang Chunyou. Antiproliferative effect of resveratrol in pancreatic cancer cells. Phytother Res. 2010;24(11):1637-44. doi: 10.1002/ptr.3157, PMID 21031621.
40. Cai, Jing, Zhao Xiang-Li, Liu An-Wen, Nian Hua, Zhang Shu-Hui. Apigenin inhibits hepatoma cell growth through alteration of gene expression patterns. Phytomedicine. 2011;18(5):366-73. doi: 10.1016/j.phymed.2010.08.006, PMID 20850954.

41. Yu, Hua, Richard Jove Richard. The STATs of cancer- new molecular targets come of age. Nature Reviews Cancer. 2004;4(2):97-105. doi: 10.1038/nrc1275, PMID 14964307.

42. Garcia Roy, Bowman TL, Niu G, Yu H, Minton S, Muro-Cacho CA, Cox CE, Falcone R, Fairclough R, Parsons S, Laudano A, Gazit A, Levitzki A, Kraker A, Jove R. Constitutive activation of Stat3 by the Src and JAK tyrosine kinases participates in growth regulation of human breast carcinoma cells. Oncogene. 2001;20(20):2499-513. 10.1038/sj.onc.1204349, PMID 11420660.

43. Masuda, Muneyuki, Suzui Masumi, Lim Jin TE, Weinstein I Bernard. Epigallocatechin-3-gallate inhibits activation of HER$2 /$ neu and downstream signaling pathways in human head and neck and breast carcinoma cells. Clinical Cancer Research. 2003;9(9):3486-91. PMID 12960141.

44. Muthian, Gladson, John J. Bright John J. Quercetin, a flavonoid phytoestrogen, ameliorates experimental allergic encephalomyelitis by blocking IL-12 signaling through JAKSTAT pathway in $\mathrm{T}$ lymphocyte. J Clinical Immunology. 2004;24(5):542-52. 10.1023/B:JOCI.0000040925.55682.a5, PMID 15359113.

45. Pratheeshkumar Poyil, Sreekala Chakkenchath, Zhang Zhuo, Budhraja Amit, Ding Songze, Son Young-Ok, Wang Xin. Cancer prevention with promising natural products: mechanisms of action and molecular targets. Anti-Cancer Agents in Medicinal Chemistry (formerly current medicinal chemistry-anti-cancer agents). 2012;10:1159-84.

46. Garnett, Mathew J, Richard Marais Richard. Guilty as charged: B-RAF is a human oncogene. Cancer Cell. 2004;6(4);313-9. doi: 10.1016/j.ccr.2004.09.022, PMID 15488754.

47. Hua Wen Feng, Fu Yong Shui, Liao Yi-Ji, Xia Wen-Jie, Chen Yang Chao, Zeng Yi-Xin, Kung Hsiang-Fu, Xie Dan. Curcumin induces down-regulation of EZH2 expression through the MAPK pathway in MDA-MB-435 human breast cancer cells. Eur J Pharmacol. 2010;637(1-3):16-21. 10.1016/j.ejphar.2010.03.051. PMID 20385124.

48. Wu, Chia Chieh, Cheng Chun Hsiang, Lee Yi-Hui, Chang Ing-Lin, Chen Hsin-Yao, Hsieh Chen-Pu, Chueh Pin-Ju. Ursolic acid triggers apoptosis in human osteosarcoma cells via caspase activation and the ERK1/2 MAPK pathway. Journal of Agricultural and Food Chemistry. 2016;64(21):4220-6. doi: 10.1021/acs.jafc.6b00542, PMID 27171502.

49. Chaturvedi MM, Sung B, Yadav VR, Kannappan R, Aggarwal BB. $\mathrm{NF}-\mathrm{\kappa B}$ addiction and its role in cancer: 'one size does not fit all'. Oncogene. 2011;30(14):1615-30. 10.1038/onc.2010.566, PMID 21170083.

50. Pahl HL. Activators and target genes of Rel/NF-kappaB transcription factors. Oncogene. 1999;18(49):6853-66. doi: 10.1038/sj.onc.1203239. PMID 10602461.

51. Coope HJ, Atkinson PG, Huhse B, Belich M, Janzen J, Holman MJ, Klaus GG, Johnston LH, Ley SC. CD40 regulates the processing of NF-kappaB2 p100 to p52. EMBO J.2002;21(20):5375-85. doi: 10.1093/emboj/cdf542, PMID 12374738.

52. Manna, Sunil K, Asok Mukhopadhyay Asok, Bharat B, Aggarwal Bharat B. Resveratrol suppresses TNF-induced activation of nuclear transcription factors NF- $\mathrm{\kappa B}$, activator protein-1, and apoptosis: potential role of reactive oxygen intermediates and lipid peroxidation. Journal Immunology. 2000;164(12):6509-19. 10.4049/jimmunol.164.12.6509, PMID 10843709.

53. Divya Chandrasekhar S, Radhakrishna Pillai M. Antitumor action of curcumin in human papillomavirus-associated cells involves downregulation of viral oncogenes, prevention of $\mathrm{NFKB}$ and AP-1 translocation, and modulation of apoptosis. Molecular carcinogenesis: published in cooperation with the University of Texas M D Anderson Cancer Center. 2006;45:320-32. 